\title{
The COVID-19 crisis will exacerbate maternal and child undernutrition and child mortality in low- and middle-income countries
}

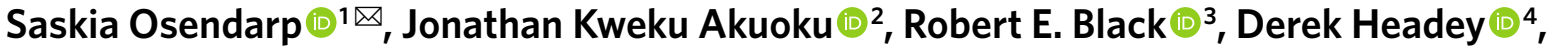 \\ Marie Ruel ${ }^{4}$, Nick Scott ${ }^{5}{ }^{5}$, Meera Shekar ${ }^{2}$, Neff Walker ${ }^{3}$, Augustin Flory ${ }^{6}$, Lawrence Haddad7, \\ David Laborde $\mathbb{D}^{4}$, Angela Stegmuller $\mathbb{B}^{3}$, Milan Thomas $\mathbb{D}^{6}$ and Rebecca Heidkamp ${ }^{3}$
}

The economic crisis and food and health system disruptions related to the COVID-19 pandemic threaten to exacerbate undernutrition in low- and middle-income countries (LMICs). We developed pessimistic, moderate and optimistic scenarios for 2020-2022 and used three modelling tools (MIRAGRODEP, the Lives Saved Tool and Optima Nutrition) to estimate the impacts of pandemic-induced disruptions on child stunting, wasting and mortality, maternal anaemia and children born to women with a low body mass index (BMI) in 118 LMICs. We estimated the cost of six nutrition interventions to mitigate excess stunting and child mortality due to the pandemic and to maximize alive and non-stunted children, and used the human capital approach to estimate future productivity losses. By 2022, COVID-19-related disruptions could result in an additional 9.3 million wasted children and 2.6 million stunted children, 168,000 additional child deaths, 2.1 million maternal anaemia cases, 2.1 million children born to women with a low BMI and US\$29.7 billion in future productivity losses due to excess stunting and child mortality. An additional US\$1.2 billion per year will be needed to mitigate these effects by scaling up nutrition interventions. Governments and donors must maintain nutrition as a priority, continue to support resilient systems and ensure the efficient use of new and existing resources.

E ven before the COVID-19 pandemic hit, the world was already not on track to achieve Sustainable Development Goal 2-to eliminate hunger and all forms of malnutrition by $2030^{1}$. The pandemic and related global economic recession are severe setbacks to already insufficient progress towards meeting the global nutrition targets set for 2025 for stunting, wasting, maternal anaemia and breastfeeding ${ }^{1}$. Pandemic-related economic contractions and disruptions to food and health systems now threaten to exacerbate maternal and child undernutrition across low- and middle-income countries (LMICs) $)^{2}$.

Measuring the effects of COVID-19 disruptions on the delivery of essential health and nutrition interventions has proven challenging, as resilient, real-time information systems were not well established in many countries before the crisis ${ }^{3}$. However, several efforts from major organisations have begun to yield some evidence. The World Health Organization's Pulse Survey on Continuity of Essential Health Services During the COVID-19 Pandemic reported health service disruptions between March and June 2020 in $90 \%$ of the 105 countries surveyed across five regions. More than half of the surveyed countries documented disruptions in antenatal care, sick child services and management of malnutrition in 2020, and $70 \%$ reported disruptions in routine immunization ${ }^{4}$. Similar findings were reported in January 2021 by UNICEF, including a $30 \%$ overall reduction in the coverage of essential nutrition services, including school feeding, micronutrient supplementation and nutrition promotion programmes in LMICs, as well as programmes for the treatment of severe wasting in children in $2020^{5}$. The World Bank's high-frequency monitoring phone surveys also found that a large percentage of households surveyed in four African countries reported not having received medical attention during the pandemic owing to fear of contracting the virus or government restrictions on mobility and social distancing requirements ${ }^{3}$. Experience from previous pandemics suggests that health system recovery could be slow; for example, it took more than a year for health care services to recover fully after the 2014 Ebola outbreak in West Africa ${ }^{6}$.

Evidence on the economic and food security impacts of COVID-19 is likewise beginning to emerge. Global estimates from mid-2020 (economic growth forecasts have since worsened) suggested that economic contractions and food supply chain disruptions had led to 95 million people falling into extreme poverty and had contributed to increased household food insecurity ${ }^{7,8}$. Updated projections from the World Bank suggested that, ultimately, up to 150 million additional people would be pushed into extreme poverty in 2020 as a result of the pandemic ${ }^{9}$. Evidence from household phone surveys in Asia and Africa also points to disturbingly large increases in poverty and food insecurity throughout $2020^{10-14}$. Disruptions in supply chains for perishable nutrient-rich foods, such as fruits and vegetables and animal-sourced foods, have been reported at local levels, especially during lockdowns, leading to price volatility and declining consumption of these foods ${ }^{8,15}$. The pandemic and pandemic response have resulted in millions of people losing their sources of income. To cope with this, households have shifted to less expensive sources of calories, including starchy staples, cereals, oils and/or non-perishable ultra-processed foods, and reduced their consumption of nutrient-rich fruit and vegetables and animal-sourced foods, such as dairy, meat and fish ${ }^{13,16,17}$.

'Micronutrient Forum, Washington, DC, USA. ${ }^{2}$ World Bank, Washington, DC, USA. ${ }^{3}$ Johns Hopkins Bloomberg School of Public Health, Baltimore, MD, USA. ${ }^{4}$ International Food Policy Research Institute (IFPRI), Washington, DC, USA. ${ }^{5}$ Burnet Institute, Melbourne, Victoria, Australia. ${ }^{6}$ Asian Development Bank, Washington, DC, USA. ${ }^{7}$ Global Alliance for Improved Nutrition (GAIN), Geneva, Switzerland. ${ }^{凶}$ e-mail: saskia.osendarp@micronutrientforum.org 
These shifts probably lead to poorer-quality diets, which can in turn increase the risks of undernutrition, especially micronutrient deficiencies. Social protection programmes, including cash and food transfers, and school meals were disrupted early in the pandemic $^{18}$, and although many countries have adapted, modified or later scaled up their programmes ${ }^{19,20}$, these are likely to fall short of needs and to be insufficient to sustain pre-crisis living standards $^{14}$. Comparisons with historical evidence on the impact of the pandemic on poverty and food security are challenging, due to the unprecedented nature and scale of the current pandemic. However, the 2007-2008 world food price crisis showed how global trade shocks can translate into volatility in domestic food markets, resulting in longer-term declines in the consumption of nutrient-dense foods and a worsening of nutritional status and health for the most vulnerable populations ${ }^{21}$.

Early insights into the disruptions to economic, food and health systems suggest a range of potential implications for nutri$\operatorname{tion}^{22}$. There have been separate estimates of the impacts of health system shocks on child wasting and maternal and child mortality ${ }^{23,24}$ and of the economic shocks on poverty in the early stages of the pandemic ${ }^{11-13,15}$. To date, however, there has been no comprehensive assessment of the combined effects of the disruptions to economic, food and health systems on maternal and child undernutrition.

The aim of this paper is to fill in some of these gaps using several modelling tools (that is, the MIRAGRODEP computable general equilibrium model, Lives Saved Tool (LiST) and Optima Nutrition model) to present a multi-year picture of the potential consequences of the pandemic-triggered economic, food and health systems crisis for maternal and child nutrition in 118 LMICs and the cost of interventions to mitigate these impacts. We estimated the potential impacts under optimistic, moderate and pessimistic scenarios for the 3-year period of 2020-2022 on: (1) child stunting, wasting and mortality, maternal anaemia and children born to women with a low body mass index (BMI); and (2) associated human productivity losses due to longer-term impacts of early life malnutrition, including lost years of schooling and economic productivity ${ }^{25}$. Mitigating the impact of these disruptions requires extra financial resources, so we also estimated the effects of the pandemic on: the domestic and donor financing landscape for nutrition; the additional costs of interventions to mitigate the rise in maternal and child undernutrition; and the potential benefits of optimizing budget allocations for nutrition.

\section{Results}

Maternal and child undernutrition and child mortality. Child wasting. Based on predicted declines in gross national income (GNI) in the 118 included countries included in the study, and the relationship between the GNI and the national prevalence of wasting, the number of children under 5 years with wasting (weight-for-height $z$ score (WHZ) $<-2$ s.d.) in 2020-2022 will increase in the moderate scenario by an additional 9.3 million (optimistic scenario $=6.4$ million; pessimistic scenario $=13.6$ million) (Table 1 ). Two-thirds of these additional wasted children in the moderate scenario will be in South Asia (6.2 million) and one-fifth (1.9 million) will be in sub-Saharan Africa.

Child stunting. In the moderate scenario, an estimated 2.6 million additional children (optimistic $=1.5$ million; pessimistic $=3.6$ million) will be stunted in 2022 compared with 2019 owing to interruptions in nutrition services and deteriorations in household poverty status (Table 1). Of these, almost 1.2 million will be in sub-Saharan Africa and 790,000 will be in South Asia (Supplementary Table 1).

Mortality in children under 5 years of age. In the moderate scenario, there will be roughly 168,000 additional deaths of children

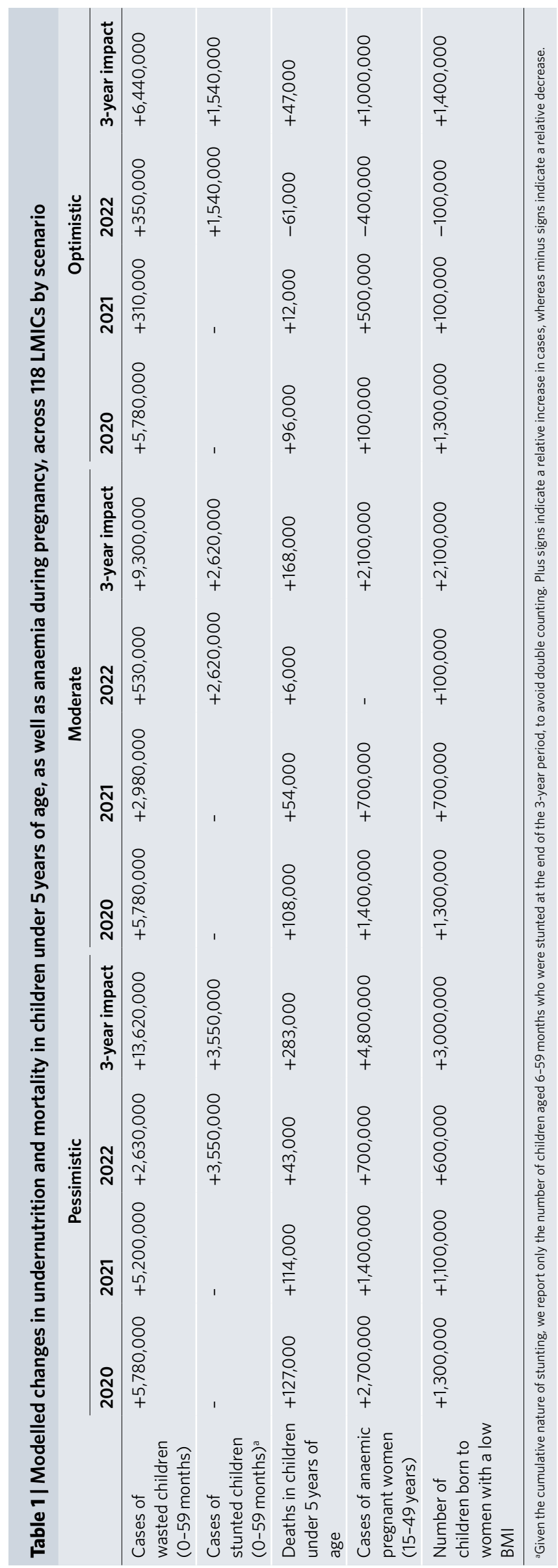


under 5 years (optimistic $=47,000$; pessimistic $=283,000$ ) in the 118 surveyed countries in 2020-2022 due to predicted increases in child wasting and declines in nutrition intervention coverage (Table 1). Consistent with wasting, most of these additional deaths will be in South Asia and sub-Saharan Africa (Supplementary Table 1). However, under the optimistic scenario, a $10 \%$ increase in the treatment of moderate acute malnutrition (MAM) in 2022 relative to 2019 would contribute to 61,000 lives saved in 2022 and a net reduction of 47,000 deaths over the 3 years (Table 1 ).

Maternal anaemia and low BMI. The moderate scenario projects there will be an additional 2.1 million pregnant women with any form of anaemia (optimistic $=1.0$ million; pessimistic $=4.8$ million) in the 118 countries in 2020-2022 compared with 2019 (Table 1). Furthermore, an additional 2.1 million children will be born to women with a low BMI (optimistic $=1.4$ million; pessimistic $=3.0$ million) in 2020-2022 (Supplementary Table 1).

Adverse birth outcomes. Two maternal nutrition interventions introduced in 2022 under the optimistic scenario would have a positive impact on small-for-gestational-age and preterm births and stillbirths: multiple micronutrient supplements (MMS) in place of iron-folic acid (IFA); and balanced energy and protein supplementation for malnourished pregnant women. A 10-percentage-point increase in coverage for both interventions in 2022 would prevent an estimated 400,000 cases of small-for-gestational-age births, 296,000 preterm births and 7,600 stillbirths across the 118 countries (Supplementary Table 1).

Future productivity losses. The estimated additional burden of childhood stunting and child mortality would result in future productivity losses (between the ages of 18 and 65 years, using country-specific life expectancy) of US $\$ 14.9$ billion, US $\$ 29.7$ billion and US $\$ 44.3$ billion under the optimistic, moderate and pessimistic scenarios, respectively. Additional cases of anaemia during pregnancy would result in US\$79 million in lost productivity (optimis$\mathrm{tic}=\mathrm{US} \$ 36$ million; pessimistic $=$ US $\$ 177$ million) in $2020-2022$ (Fig. 1). Across the three outcomes, these losses represent between 0.1 and $0.3 \%$ of current GNI in the 118 sample countries.

Financing landscape for nutrition. Using the MIRAGRODEP moderate scenario, our dynamic model implies that official development assistance (ODA) to nutrition-specific and -sensitive sectors will decline slightly through 2021 before a lagged effect fully manifests in 2022 with a decline of $8.9 \%$. Under this scenario, ODA would not recover to pre-crisis levels until 2028. Domestic financing for health (including nutrition-specific financing) is projected to fall (or have fallen) by $7.2 \%$ in $2020,4.2 \%$ in 2021 and $2.2 \%$ in 2022, and will not recover to pre-crisis levels until the end of the decade unless there is strong recovery of economic growth in LMICs. Relative to no-COVID projections, all three disruption scenarios project a significant ODA shortfall to nutrition-specific and -sensitive sectors through 2030 (pessimistic $=19 \%$; moderate $=14 \%$; optimistic $=9 \%$ ), accompanied by a similar decrease in domestic health budgets. While adding the debt-to-gross domestic product (GDP) ratio as an explanatory variable does not change these projections significantly (the debt ratio and GDP growth are strongly correlated in the study years), the model may underestimate the impact of the crisis on nutrition financing, because debt levels in the aftermath of the global crisis are expected to reach levels not observed over the past two decades (Supplementary Methodology 2). In contrast, it remains to be seen what mitigating effects debt forgiveness and other global developments (innovative financing and new commitments made at the 2021 nutrition summits) that cannot be accounted for in our panel regressions may have on nutrition financing.

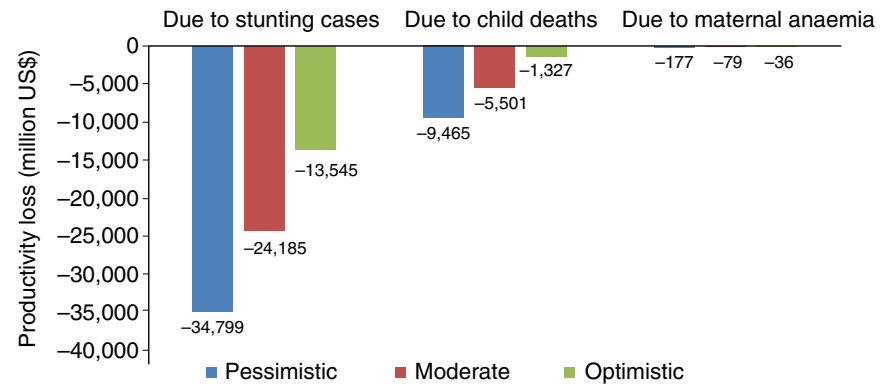

Fig. 1 | Future productivity losses due to additional cases of child stunting, mortality and maternal anaemia. To estimate lost productivity from nutrition-related impacts, the human capital approach was used. This involved calculating the present value of future productivity based on potential wages and potential time in the workforce, as outlined in the Methods.

\section{Additional financing needs and optimizing existing financing.} Based on the projected increases in stunting, wasting and anaemia during pregnancy in the moderate scenario, we estimate that an additional US $\$ 1.2$ billion per annum (optimistic $=$ US $\$ 763$ million; pessimistic $=$ US $\$ 1.7$ billion) will be needed to mitigate the impacts of COVID-19 on maternal and child undernutrition (Table 2). Scaling up nutrition interventions with direct and indirect effects on child mortality will also prevent additional child deaths.

Improvements in the allocative efficiency of six nutrition interventions (Fig. 2) could avert some of the pandemic's indirect effects on nutrition. Our multi-country analysis suggests that realigning financing from the provision of complementary foods towards a more targeted and balanced mix of interventions could lead to as many as 8.2 million (4.9\%) fewer stunted children under 5 years of age in 2022 and 339,000 (2.2\%) deaths in children under 5 years averted in 2020-2022 compared with a scenario without optimization (Fig. 2). The illustrative intervention mix includes an expansion of severe acute malnutrition (SAM) treatment and infant and young child feeding counselling for children between 6 and 23 months of age in food-secure populations, combined with provision of complementary foods in food-insecure populations, increases in vitamin A supplementation, breastfeeding promotion and balanced energy and protein supplementation to women with a low BMI.

\section{Discussion}

While women of reproductive age and young children are largely spared COVID-19's direct effects (that is, serious disease and death), our projections demonstrate that, regardless of the scenario, the COVID-19 crisis is expected to have dramatic indirect effects on maternal and child undernutrition and child mortality in the current generation. The disruptions to health and food systems caused by the pandemic, and especially the global economic recession it has triggered, will probably continue at least until the end of 2022, jeopardizing the efforts of both LMICs and donors to achieve global nutrition targets and Sustainable Development Goal 2. The nutritional impacts of the COVID-19 crisis could have massive, long-term productivity consequences that could extend to future generations. Poor nutrition during early life stunts both physical and cognitive development, affects schooling performance and adult productivity, increases the risks of overweight/obesity and diet-related non-communicable diseases later in life, and triggers the intergenerational transmission of malnutrition ${ }^{25}$. These longer-term and irreversible impacts of early life malnutrition on human capital formation lead to very high estimated returns on investment (ROIs), up to US\$35 for every dollar spent ${ }^{26}$. This is one of the highest ROIs in development and it compares favourably with the ROIs of other global health initiatives, including recent 
estimates for childhood vaccinations (US\$21 ROI). In addition, there could be immediate impacts on the effectiveness of pandemic mitigation efforts in populations with high rates of undernutrition. The association between undernutrition and reduced immune function is well documented, and undernutrition during pregnancy and early childhood is known to impair the immune respons $\mathrm{e}^{27}$ and possibly also the response to vaccinations ${ }^{28}$.

If we consider the moderate scenario used in our analyses, the changes in GNI per capita due to the COVID-19 crisis could result in an additional 9.3 million wasted children between 2020 and 2022-quite a large increase relative to the 47 million wasted children reported in $2019^{29}$. Reductions in the coverage of nutrition services and increases in household food insecurity could add 2.6 million stunted children by 2022 to the estimated 144 million in 2019. After two decades of global decline in stunting, this would effectively reverse the past year or two of progress. Under the moderate scenario, these increases in child undernutrition and declines in the coverage of essential nutrition services are expected to lead to roughly 168,000 additional deaths of children under 5 years by 2022; and the increases in child stunting and mortality are expected to lead to future productivity losses of US $\$ 29.7$ billion-enough to impact national economies.

Our estimates for increased stunting may be conservative. First, to avoid double counting due to the progressive and cumulative nature of child stunting, we calculated estimates for stunting in children under 5 years of age only in 2022-the final year of our projections. These estimates therefore exclude additional stunting cases that developed among children who were under 5 years of age in 2020-2021 but older than 5 years of age in 2022 . Second, the projected increases in maternal anaemia (2.1 million cases by 2022) and children born to women with a low BMI (3 million by 2022) suggest that stunting may increase further after 2022, as poor maternal nutritional status is a major risk factor for adverse birth outcomes and subsequent child malnutrition ${ }^{30}$. Third, all of our estimates used the 2019 prevalence data as a baseline, not taking into account that without COVID-19 the world may have seen similar rates of improvement in stunting in 2020 as those observed before 2019.

The projected deteriorations in nutritional status, child mortality and productivity are sobering and demand immediate cross-sector action. The optimistic scenario signals the potential for the health sector to mitigate short- and long-term negative impacts by focusing on rapid recovery and expanding the coverage, even modestly, of essential maternal and child nutrition services, including the management and treatment of MAM. The optimistic scenario further suggests that this crisis could be used as an opportunity to accelerate the introduction and scale up of two maternal nutrition interventions-providing pregnant women with MMS (rather than IFA) and providing low-BMI pregnant women with balanced energy and protein supplements. United Nations agencies have specifically called for the provision of MMS to pregnant women to ensure adequate micronutrient intake during the COVID-19 crisis $^{31}$. Such an effort would follow an earlier example of using a crisis to scale up a new intervention-the introduction of multiple micronutrient powder during the 2006 tsunami response in Aceh, Indonesia ${ }^{32}$. Similarly, countries could consider introducing small-quantity lipid nutrient supplements as part of their COVID-19 response strategies, given the promising evidence that they may improve child growth ${ }^{33}$ and reduce the risk of child mortality ${ }^{34}$.

Our costing and optimization estimates illustrate that allocative efficiency gains can be achieved using existing nutrition resources. It is notable that breastfeeding promotion (which reduces mortality) and improved complementary feeding (which reduces stunting) would also serve as double-duty actions that could help to reduce both obesity and micronutrient deficiencies ${ }^{35}$. Further improvements may be possible through technical efficiency gains, such as reducing the cost of SAM treatment or, as noted previously,
Table 2 | Additional per-annum cost of interventions to mitigate COVID-related increases in malnutrition outcomes under various modelled scenarios

\begin{tabular}{llll} 
Outcome & Pessimistic & Moderate & Optimistic \\
\hline $\begin{array}{l}\text { Stunting } \\
\text { (0-59 months) }\end{array}$ & $1,258,000,000$ & $930,000,000$ & $545,000,000$ \\
$\begin{array}{l}\text { Wasting } \\
\text { (0-59 months) }\end{array}$ & $454,000,000$ & $310,000,000$ & $215,000,000$ \\
\hline $\begin{array}{l}\text { Maternal anaemia } \\
\text { (15-49 years) }\end{array}$ & $14,000,000$ & $6,000,000$ & $3,000,000$ \\
\hline Total & $1,726,000,000$ & $1,246,000,000$ & $763,000,000$ \\
\hline
\end{tabular}

All values are given in US\$. Additional resources needed to address expected increases in stunting wasting and maternal anaemia were calculated by multiplying the additional burden of each outcome (estimated using the LiST) by the cost of each case of stunting averted or severe wasting treated, as outlined in Supplementary Methodology 2.

switching from IFA to MMS during pregnancy. However, our findings should not be interpreted as prescriptive at the country level. While it is not clear how feasible allocative efficiency gains would be during a crisis situation such as the COVID-19 pandemic, our analysis demonstrates that this is an avenue worth exploring to save lives and reduce stunting, especially when financing is constrained. The optimal results and allocative efficiency gains will vary across countries, depending on demographics, epidemiological factors and baseline intervention coverages, as well as context-specific costs, priority targets, delivery platforms and other constraints. Therefore, while these global estimates demonstrate that large efficiency gains are feasible, countries need customized analyses to help decide how to deliver more nutrition for the money available.

To mitigate the effects of COVID-19 on child stunting, child wasting and maternal anaemia, we estimated that at least an additional US\$762 million to US\$1.7 billion per annum will be needed to achieve the global nutrition targets on top of the additional US $\$ 7$ billion per annum estimated in 2017. While there may be some deferred costs that were not incurred in 2020, even before COVID-19, funding was well short of what was needed. Furthermore, these deferred costs would be insufficient to address the additional cases of adverse nutritional outcomes. Previous evidence shows that before COVID-19, ODA flows towards nutrition-specific and -sensitive sectors were already substantially below recommended levels of donor financing ${ }^{1}$. Our results offer a conservative estimate given that they are restricted to child wasting and stunting and maternal anaemia, and cover only six nutrition-specific interventions using pre-COVID-19 cost assumptions. Critically, recent evidence from countries that have achieved notable improvements in childhood stunting reinforce that, for stunting reduction, policies and interventions that address the economic and food systems drivers of nutrition are just as important as those that address health determinants ${ }^{36,37}$. The critical actions across health, food and social protection systems that countries should prioritize to mitigate the potentially devastating impacts of the pandemic on maternal and child nutrition have recently been described ${ }^{38}$ and are summarized in the Supplementary Discussion.

It is important to note that our estimates, based on the 2020 projected longer-term impacts of the COVID-19 crisis on food systems, health systems, economies and ODA worldwide, are to be interpreted as projections. They do not document actual changes. The projections also do not incorporate the effects of government interventions and other mitigation measures implemented at the country level, or renewed policy commitments to invest in nutrition as a result of the pandemic. It is reassuring to see that governments have indeed implemented various mitigation and adaptation measures to ensure the safe delivery of essential nutrition services, 


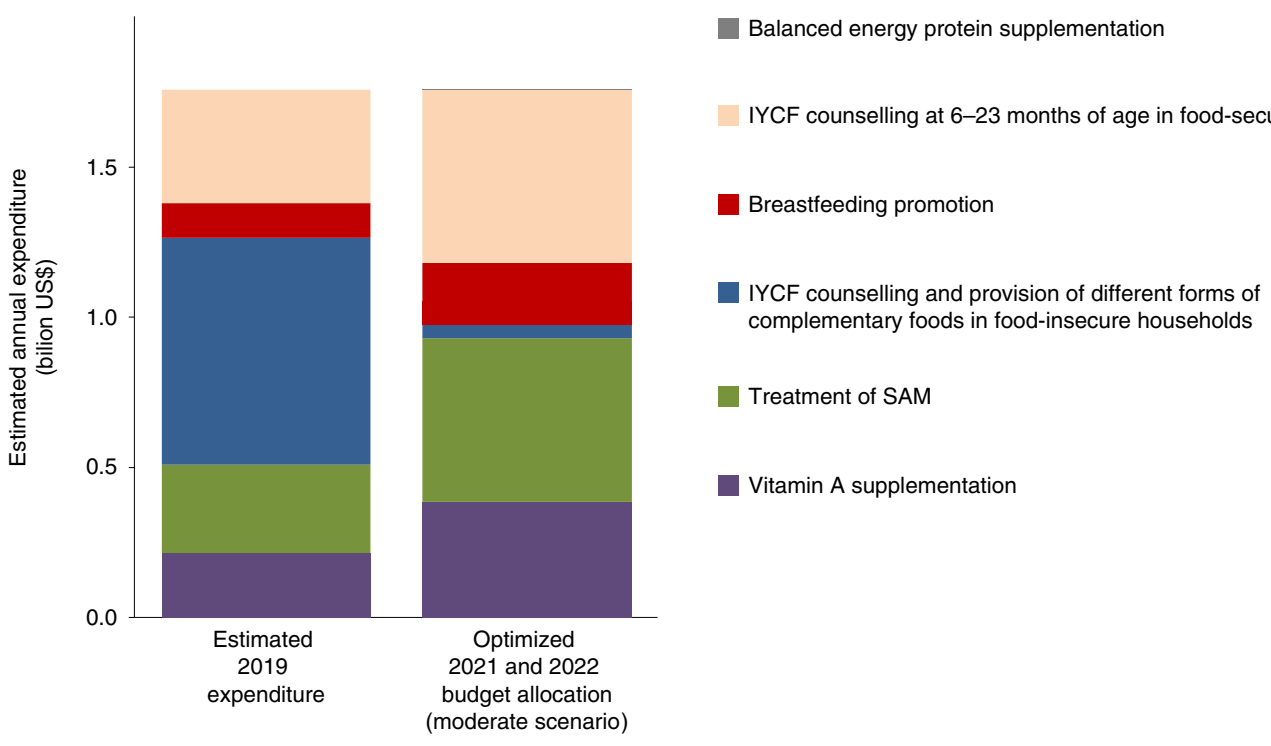

Fig. 2 | Optimization of interventions to reduce child stunting and child mortality. Left bar: estimated expenditure on selected nutrition interventions in 2019, calculated by combining coverage estimates (moderate scenario) and unit cost estimates. Right bar: optimized budget allocation to maximize alive and non-stunted children, with the total funding envelope estimated from ODA projections relative to 2019. The results have been aggregated over 118 countries, and variations exist across countries due to demographic, epidemiological and economic factors. Breastfeeding promotion includes both early initiation and exclusive breastfeeding promotion. IYCF, infant and young child feeding.

maintain adequate food supply and offset losses in income and widespread food insecurity. Examples of strategies that countries have adopted to prevent an interruption of essential health and nutrition services include the use of digital health technologies, mobile medical teams or campaigns (for example, vaccination campaigns), task shifting and additional staff training, home delivery of food and supplements, transport/financial support to patients, and strengthening of community outreach networks ${ }^{4,39}$. To maintain an adequate food supply, countries are exploring interventions to improve short- to medium-term agricultural production ${ }^{40,41}$, while government programmes working with small and medium enterprises have supported the adoption of new marketing, sales and distribution strategies as a result of disruptions in value chains ${ }^{39}$. To mitigate the impacts of the economic crisis on income losses and food security, governments have implemented, adapted or scaled up social safety net programmes, including cash and food transfers in more than 200 countries $^{14,19}$. However, while little information is available on the efficiency and effectiveness of mitigation measures in health, food and social protection systems, recent data from 16 household surveys in nine LMICs suggest that these measures may have been insufficient to prevent increases in poverty and household food insecurity ${ }^{14}$. Moreover, it is likely that these interventions stretch government budgets, fail to meet the overwhelming need for these interventions, given the devastating impact of COVID-19 on poverty levels, and leave behind many of the most vulnerable, including women, migrant and refugee populations and the ultra-poor, among others ${ }^{42}$. Future analyses from this group will further explore how programme adjustments may have affected the impact of COVID-19 on nutrition across countries.

Our analysis has some limitations. First, uncertainty is present in all modelling exercises. To reflect this uncertainty, pessimistic and optimistic scenarios were developed for 2021 and 2022 based on country differences in economic forecasts and pre-COVID-19 intervention coverage. The assumptions used for the scenarios were derived from country-specific input data and observations on coverage reductions of essential health and nutrition services during previous, similar crises $^{23}$. However, the scenarios assume that all countries follow optimistic, medium or pessimistic paths, while in practice, at any given time during the pandemic, countries may have been following one path or another, and also experience sudden reversals. Given recent developments, including the rapid spread of aggressive new variants of the COVID-19 virus, it is possible that impacts on nutrition align more closely with our pessimistic scenario. In contrast, it is also possible that effective mitigation measures, accelerated vaccinations and recovery efforts will help to prevent further deterioration, in which case our optimistic scenario may be closer to reality. At this time, it is impossible to rigorously assess the accuracy of our mortality and undernutrition projections, even for 2020, given the lack of current nationally representative data on maternal and child nutrition or mortality outcomes.

Second, there are potentially strong assumptions involved in linking together different kinds of economic and health models. We assume that historical linkages between economic growth shocks and nutrition or health services and nutrition still apply today. We also assume that economic shocks and health service shocks independently and additively affect nutrition outcomes. However, we cannot rule out that our aggregate estimates may be biased upwards or downwards, depending on the nature of the interaction between economic and health service shocks.

Third, the paucity of pre-COVID-19 baseline data on dietary intake and micronutrient status across LMICs precluded modelling the effects of the pandemic on these other forms of malnutrition. However, the reported disruptions in supply chains for perishable nutrient-rich foods and related drops in availability, affordability and consumption ${ }^{43}$ suggest potentially large impacts on micronutrient deficiencies and possible increases in obesity ${ }^{44}$. These limitations suggest that future research should focus on improving both data availability (for example, micronutrient deficiencies) and the integration of economic and health/nutrition models.

In conclusion, the COVID-19 pandemic has created a nutritional crisis in LMICs, Without swift and strategic responses by subnational, national, regional and international actors, COVID-19 will not only reverse years of progress and exacerbate disparities in disease, malnutrition and mortality, but will also jeopardize human capital development and economic growth for the next generation ${ }^{25}$ Our projections on the vast and irreversible short- and long-term 
Table 3 | Disruption scenarios reflecting the effects of the COVID-19 crisis on GNI and nutritional intervention coverage in LMICs

\begin{tabular}{|c|c|c|c|c|c|c|c|c|c|}
\hline & \multicolumn{3}{|c|}{2020} & \multicolumn{3}{|c|}{2021} & \multicolumn{3}{|c|}{2022} \\
\hline & Pessimistic & Moderate & Optimistic & Pessimistic & Moderate & Optimistic & Pessimistic & Moderate & Optimistic \\
\hline $\begin{array}{l}\text { (mean) } \\
\text { Coverage change relative to the country-snecific haseline (nre-coVID-19) by nutrition intervention }\end{array}$ & $-6.1 \%$ & $-6.1 \%$ & $-6.1 \%$ & $-5.4 \%$ & $-3.4 \%$ & $-0.47 \%$ & $-2.9 \%$ & $-0.73 \%$ & $-0.45 \%$ \\
\hline Breastfeeding promotion & $-42.3 \%$ & $-22.8 \%$ & $-14.3 \%$ & $-21.2 \%$ & $-11.4 \%$ & $-7.2 \%$ & $-10.6 \%$ & $0 \%$ & $+5.0 \%$ \\
\hline $\begin{array}{l}\text { Complementary feeding SBCC } \\
\text { (food secure) }\end{array}$ & $-42.3 \%$ & $-22.8 \%$ & $-14.3 \%$ & $-21.2 \%$ & $-11.4 \%$ & $-7.2 \%$ & $-10.6 \%$ & $0 \%$ & $+5.0 \%$ \\
\hline Vitamin A supplementation & $-42.3 \%$ & $-22.8 \%$ & $-14.3 \%$ & $-21.2 \%$ & $-11.4 \%$ & $-7.2 \%$ & $-10.6 \%$ & $0 \%$ & $+5.0 \%$ \\
\hline Treatment of SAM & $-50.0 \%$ & $-25.0 \%$ & $-15.0 \%$ & $-25.0 \%$ & $-12.5 \%$ & $-7.5 \%$ & $-12.5 \%$ & $0 \%$ & $+5.0 \%$ \\
\hline IFA in pregnancy & $-51.9 \%$ & $-26.9 \%$ & $-18.5 \%$ & $-25.9 \%$ & $-13.4 \%$ & $-9.2 \%$ & $-13.0 \%$ & $0 \%$ & $0 \%^{\mathrm{a}}$ \\
\hline $\begin{array}{l}\text { Multiple micronutrients in } \\
\text { pregnancy }\end{array}$ & - & - & - & - & - & - & - & - & $+5.0 \%{ }^{\mathrm{a}}$ \\
\hline
\end{tabular}

Justifications for the magnitude of the changes are outlined in the Methods. Minus symbols indicate a percentage decrease, whereas plus symbols indicate a percentage increase. aln 2022 , the optimistic scenario for IFA is replaced by multiple micronutrient supplementation, and coverage is increased relative to the IFA baseline. ${ }^{b}$ For MAM treatment in children under 5 years of age, an increase to $10 \%$ coverage in 2022 is assumed for all 118 countries. Before COVID-19, 36 countries reported MAM programmes. SBCC, social and behaviour change communication.

implications of the COVID-19 crisis on nutrition are meant to inform decision-makers on the urgent need to step up nutrition investments as part of COVID-19 responses. We must act now to strengthen the delivery of policies and both direct and indirect nutrition interventions across health, food and social protection systems, both in the immediate and long term. Additional ODA and domestic funding, as well as better use of existing resources, will be critical to mitigating the damage from the pandemic on lives lost and human capital, to build up populations' resilience for future similar shocks and to reinvigorate efforts to achieve global development targets and safeguard good nutrition for all, now and in the future.

\section{Methods}

Estimating effects on maternal and child undernutrition and child mortality.

To estimate the overall indirect effects of COVID-19 on maternal and child undernutrition and child mortality, and to explore possible mitigation strategies, we used a five-step process across three assumption scenarios: pessimistic, moderate and optimistic (Table 3).

In the first step, we estimated changes in GNI per capita and poverty (the percentage of the population living on $<$ US $\$ 1.90 \mathrm{~d}^{-1}$ ) due to COVID-19 disruptions during 2020, 2021 and 2022 in 118 LMICs, using three different economic recovery scenarios. Second, we translated these different projections for GNI per capita into country-specific changes in the prevalence of different levels of child wasting and maternal low BMI status using a recent statistical analysis that linked growth shocks to wasting and low BMI risks. Third, we entered the poverty and wasting estimates, along with assumptions about levels of disruption to health and nutrition services, into the LiST to estimate changes in mortality and stunting in children under 5 years and maternal anaemia. Finally, we used the Optima Nutrition model to explore possible strategies to mitigate the predicted increases in undernutrition and child mortality. The methods and inputs for each of these steps are detailed below.

For the first step, we used MIRAGRODEP-a global computable general equilibrium model linked to country-specific household survey data-to predict the effects of COVID-19 disruptions on GNI per capita, household incomes and US $\$ 1.90 \mathrm{~d}^{-1}$ poverty rates between 2020 and $2022^{45}$. The MIRAGRODEP modelling systematically structures economic disruption scenarios using a wide range of available country-level data on impacts on international trade, finance, consumer mobility and government fiscal policies. The economic channels of disruption included changes in labour force participation and labour productivity due to lockdown policies, increased morbidity and mortality, reduced efficiency of domestic and international transportation of goods, reduced consumer demand for in-person services (for example, restaurants) and international shocks to trade and financial flows (for example, reduced demand for oil). For these analyses, country-level economic disruption data updated to October 2020 were used, thereby taking into account how countries had thus far been affected by the direct and indirect consequences of COVID-19 and resulting economic losses.

For 2020, we modelled a single GNI scenario, as there is less uncertainty on the extent of economic disruption. MIRAGRODEP results for 2020 were also checked against the most recent GDP growth estimates from various international agencies (for example, the International Monetary Fund, World Bank and Asian Development Bank) to ensure that MIRAGRODEP estimates were largely in accordance with other estimates, especially for countries with high burdens of undernutrition ${ }^{46,47}$. However, for 2021 and 2022, we opted to model three different scenarios for economic recovery from the pandemic ${ }^{48}$. The optimistic scenario assumed a fast $\mathrm{V}$-shaped economic recovery, with economic activity accelerating quickly from 2021 onwards. For example, substantial acceleration in vaccine rollouts could accelerate economic recovery. The moderate scenario assumed a second major infection wave into 2021, resulting in a stop-start $\mathrm{W}$-shaped recovery, but also high vaccine access and stronger recovery by 2022 . The pessimistic scenario assumed a protracted U-shaped recovery, with continued economic disruptions in 2021 and most countries not returning to pre-COVID-19 per-capita income levels by 2022 (Table 3). For this analysis, the magnitude of macroeconomic disruption for the three scenarios was measured as the percentage difference between GNI per capita with and without COVID-19, while impacts on poverty-also used as a proxy for food insecurity (see below) - were measured as the difference in US $\$ 1.90 \mathrm{~d}^{-1}$ poverty rates with and without COVID-19. Although MIRAGRODEP is a global model, all GDP growth and poverty change estimates are based on country-specific disruption scenarios and their endogenous economic effects (for example, price, wage and income adjustments).

Second, we used elasticities from a recent study ${ }^{49}$ that linked economic growth (GNI) shocks to child wasting and maternal low BMI risks to generate expected changes in wasting and low-BMI prevalence. Specifically, that study linked 177 demographic and health surveys from 52 LMICs to national-level GNI growth shocks and uncovered statistically significant negative relationships between changes in GNI per capita and: (1) changes in the prevalence of any (WHZ $<-1$ s.d.), moderate/severe (WHZ $<-2$ s.d.) and severe (WHZ $<-3$ s.d.) wasting among children aged 0-59 months; and (2) the prevalence of low BMI $\left(<18.5 \mathrm{~kg} \mathrm{~m}^{-2}\right)$ among mothers aged $15-49$ years. To estimate pandemic-induced increases in the number of children with wasting and women with low BMI, we applied the corresponding elasticities from that study to country-specific GNI growth projections from MIRAGRODEP, country-specific population projections, and baseline undernutrition estimates from LiST

Finally, we used LiST to estimate the degree to which interruptions in nutrition intervention delivery through health systems (Table 3) would affect the number of children under 5 years of age with stunting (height-for-age $z$ score $<-2$ s.d.) and pregnant women with anaemia (haemoglobin $<12 \mathrm{~g} \mathrm{dl}^{-1}$ ). Stunting is a cumulative rather than episodic condition, while LiST is a cohort model; therefore, to avoid double counting the same stunted children across years, we report only the total number of stunted children in 2022 (the end of the 3-year projection period). The LiST was also used to estimate the combined effects of changes in nutrition 
intervention coverage due to health system disruptions and increased child wasting from economic disruptions on mortailty in children under 5 years of age.

The LiST includes country-specific population projections and other baseline assumptions from publicly available data sources (https://www.livessavedtool. org/country-data-pack). Our analysis covers 118 LMICs with sufficient data on the coverage of nutrition and health interventions (Supplementary Methodology 1). More than $95 \%$ of the global totals of both deaths and stunting in children under 5 years of age are found in these countries. We present estimates for all 118 LMICs, as well as regional estimates for South Asia and sub-Saharan Africa (Supplementary Methodology 1) using country-specific data on the coverage of health system interventions pre-COVID-19. These projections therefore already take into account the differences in performance of health systems that occurred among countries before the pandemic. Mean annual coverage reduction assumptions for five preventative interventions across the three scenarios are consistent with the analyses by Roberton et al. ${ }^{23}$ (Table 3 ). In brief, average coverage reductions were based on observations of coverage reductions of essential health and nutrition services during previous, similar health crises, including the Ebola crisis in 2014 in West Africa and the 2003 severe acute respiratory syndrome epidemic in Taiwan. The different scenarios were derived from these average reductions, assuming different intensities of government responses and evolutions of the pandemic. For each scenario, assumptions were developed for the four components, using the following reduction categories: none ( $0 \%$ reduction), small (5\% reduction), moderate ( $10 \%$ reduction) and large ( $25 \%$ reduction). We compared our intervention coverage disruption scenarios against the most recent evidence on actual coverage reductions in essential health and nutrition services observed in 2020, as published by UNICEF, which suggested that our scenarios are in line with actual observations. As of January 2021, a 30\% overall reduction was reported over 2020 in the coverage of essential nutrition services, including school feeding, micronutrient supplementation and nutrition promotion programmes in LMICs, as well as programmes for the treatment of severe malnutrition in children $^{5}$. We included treatment of SAM with similar coverage reduction assumptions, but did not assume changes in MAM treatment coverage except in the optimistic scenario for 2022. For household food insecurity assumptions, which influence complementary feeding interventions (public provision of complementary foods for food-insecure households versus nutrition counselling for food-secure households), we used the annualized MIRAGRODEP poverty projections $\left(<\mathrm{US} \$ 1.90 \mathrm{~d}^{-1}\right)$.

As detailed in Table 3, we assumed that service disruptions would continue after initial lockdowns but become less severe over time and, in all three scenarios, would recover in 2021 to half of the 2020 decrease. Possible sources of continuing disruptions include: (1) health workers contracting COVID-19 and/or being diverted to the care of COVID-19 patients with new virus waves; (2) reduced use of available services by the population due to the perceived risk of exposure; and (3) reduced financing over time due to economic impacts. In 2022, coverage levels are expected to remain lower than pre-COVID-19 baselines (pessimistic scenario), return to 2019 levels (moderate scenario) or increase by 5\% over 2019 levels (optimistic scenario). Furthermore, under the optimistic scenario, we estimated the impacts on adverse birth outcomes and deaths in children under 5 years of age of adding three interventions in 2022: treatment of MAM in children under 5 years of age (increased to $10 \%$ coverage); provision of MMS (rather than IFA) during pregnancy (added at $10 \%$ coverage); and provision of balanced energy and protein supplementation to pregnant women with a low BMI (added at $10 \%$ coverage).

Effects on long-term productivity. We used the human capital approach to estimate lost productivity from nutrition-related impacts of the COVID-19 pandemic. This approach calculates the present value of future productivity based on potential wages and potential time in the workforce ${ }^{50}$ and is similar to the approach adopted by Shekar et al. ${ }^{26}$ for estimating the benefits of scaling up nutrition interventions. For this study, we used GNI per capita as a measure of average future wages. Estimates of lost productivity were calculated separately for stunting, nutrition-related child mortality and maternal anaemia outcomes. Total excess cases of each outcome were based on the results of the LiST modelling previously described.

For each child death, years of potential productivity were calculated as the difference between age 18 years and the lower of either the country-specific life expectancy or age 65 years. The total number of children with potential lost productivity due to premature mortality was adjusted by the probability of death between the ages of 5 and 18 years. Each year of productivity was valued using GNI per capita (current US\$) adjusted for annual GNI growth, the percentage of lifetime earnings that could be realized ${ }^{51}$ and labour share of income. Future lost productivity was discounted at $3 \%$ per year ${ }^{52,53}$. For total lost productivity in each country, the lost productivity from each death was summed across all years of lost productivity and multiplied by the adjusted number of deaths.

Stunting is a marker of early life exposure to environmental and behavioural risks and has been shown to result in lower schooling attainment and wage earnings in adulthood and lower overall national productivity ${ }^{26}$. Lost productivity from additional stunting cases was estimated using an approach similar to that used to calculate lost productivity from child deaths. However, since stunting is expected to reduce future productive capacity for each child and not prevent future labour force participation, only the reduced productive capacity was considered as potential lost productivity. Previous research estimated that decreased cognitive development due to early childhood malnutrition marked by stunting could lead to a $21 \%$ reduction in adult earnings ${ }^{54}$. Therefore, the approach described for estimating lost productivity due to premature deaths was applied to the additional number of stunting cases and multiplied by $21 \%$ to derive an estimate for the lost productivity attributable to additional stunting cases.

Excess cases of anaemia in pregnant women were translated into lost earnings due to decreased labour productivity based on the methods described by Horton and Ross ${ }^{54}$. The level of productivity lost due to anaemia differs depending on whether employment is in heavy manual labour, light manual labour or the service sector, with attributed productivity reductions of 17,5 and $4 \%$, respectively. We determined the percentage of women engaged in each type of labour for each country from the World Development Indicators database. The shares of women employed in agriculture, industry and services were categorized as working in heavy manual labour, light manual labour and service labour, respectively. Lost productivity was calculated separately for each category of employment as a product of the per-capita GNI, number of additional cases of maternal anaemia in each year, percentage of female employment in the specific category and associated reduction in productivity in that category due to anaemia. These estimates were adjusted for female labour force participation and labour share of income. The total lost productivity for each country was the sum of the estimated productivity lost across the three employment categories and the 3 years (2020-2022) modelled, discounted at $3 \%$ per year.

\section{Effects on the financing landscape for nutrition. Since 2002, ODA to} nutrition-specific and -sensitive sectors has, on average, trended with donor country economic cycles, but with a lag. We ran regressions with GDP growth lags of up to 5 years, but only the 2-year lag was statistically significant (with an elasticity slightly greater than 1 ).

Therefore, our results, like those of Stuckler et al. ${ }^{55}$, are reported with lags of up to 2 years. For each percentage point increase in national growth, there is typically a 1.6-percentage-point increase in ODA 2 years later. For each percentage point increase in the growth of domestic financing, there is a 1.5-percentage-point increase in domestic health expenditure, with no time lag.

To project how available donor and domestic resources for nutrition are expected to change across the three disruption scenarios, we developed a modelling approach based on Stuckler et al. ${ }^{55}$. First, using Creditor Reporting System data on ODA from 2002-2018 (https://stats.oecd.org/), we ran a panel regression to estimate the elasticity of aid to nutrition-specific and -sensitive sectors with respect to donor countries' economic growth. Then, we multiplied that elasticity by the MIRAGRODEP GNI growth projections to estimate the expected levels of aid to those sectors between 2020 and 2030. We conducted an analogous procedure to project the effect of domestic economic growth on domestic government health spending, using National Health Accounts panel data from the World Health Organization's Global Health Observatory (https://www.who.int/data/gho) for the same 118 countries used in the Optima Nutrition model.

\section{Estimating additional intervention costs and optimized nutrition} budget allocations. The Investment Framework for Nutrition ${ }^{26}$ estimated that approximately US\$7 billion per annum was needed to reach the World Health Assembly targets for stunting, wasting, maternal anaemia and breastfeeding by 2025. Given the disruptions caused by the COVID-19 pandemic, we anticipate that more resources will be needed to address the increased burden of malnutrition. We calculated the additional resource needs for addressing expected increases in stunting, wasting and maternal anaemia by multiplying the additional burden of each outcome estimated from the LiST modelling by the cost of each case of stunting or anaemia averted or severe wasting treated.

We used the Optima Nutrition model ${ }^{56}$ to illustrate how 2021-2022 financing could be optimally allocated across six nutrition interventions to maximize the number of alive and non-stunted children. Optima Nutrition is a cohort model that tracks children up to age 5 years of age, categorized according to their mother's breastfeeding practices, family economic status, height-for-age (stunting) status and weight-for-height (wasting) status. Children in the model can die from a range of age-specific causes, with the relative risks of dying from each cause related to the child's breastfeeding, stunting and wasting status. Interventions can improve nutritional outcomes directly or indirectly by reducing risk factors such as birth outcomes and diarrhoea incidence. The model includes an optimisation algorithm, which can be used to incrementally shift a fixed amount of funding between interventions until it achieves a budget allocation that maximizes (or minimizes) a given objective. For this analysis, the objective of maximizing alive and non-stunted children was selected to identify a priority mix of interventions to both reduce mortality from severe wasting and reduce stunting. Additional model details are available in the user guide ${ }^{57}$ or other publications ${ }^{56,58}$.

For each of the 118 LMICs in this analysis, the total expenditure on the six nutrition interventions in 2019 and 2020 was estimated by multiplying coverage estimates from the moderate scenario (Table 3) by country-specific unit costs (Supplementary Methodology 2). Given evidence of a lagged decline in ODA starting in 2022, we assumed that the total available financing for the nutrition 
interventions in 2021 and 2022 would equal the total 2019 expenditure. The model was projected for the entire $2020-2022$ period. The pre-optimization epidemiological projections and intervention coverages were aligned with the moderate scenario (Table 3) and compared with outcomes where the funding was optimized to determine the maximum number of stunting cases and child deaths that could be averted through allocative efficiency.

Reporting Summary. Further information on research design is available in the Nature Research Reporting Summary linked to this article.

\section{Data availability}

The data that support the findings of this study have been deposited at https:// drive.google.com/drive/folders/1AVqEF7gYTGmJENlya3UbUxO6A2fKBQm6?u $\mathrm{sp}=$ sharing.

\section{Code availability}

All software (modelling tools) used in the analyses of this study have been referenced previously and are available at https://www.ifpri.org/publication/ miragrodep-model (MIRAGRODEP), https://www.livessavedtool.org/resources (LiST) and https://optimamodel.com/nutrition/ (Optima Nutrition model).

Received: 7 December 2020; Accepted: 11 June 2021; Published online: 19 July 2021

\section{References}

1. The Global Nutrition Report's Independent Expert Group. 2020 Global Nutrition Report: Action on Equity to End Malnutrition (Development Initiatives, 2020); https://globalnutritionreport.org/reports/20 20-global-nutrition-report/

2. Headey, D. et al. Impacts of COVID-19 on childhood malnutrition and nutrition-related mortality. Lancet 396, 519-521 (2020).

3. Walker, D., Krubiner, C. \& Chi, Y.-L. What Do We Really Know About COVID-19's Impact on Essential Health Services? (Center for Global Development, 2021); https://www.cgdev.org/blog/what-do-we-really-knowabout-covid-19s-impact-essential-health-services

4. Pulse Survey on Continuity of Essential Health Services During the COVID-19 Pandemic: Interim Report, 27 August 2020 (World Health Organization, 2020); https://www.who.int/publications/i/item/WHO-2019nCoV-EHS continuity-survey-2020.1

5. Nutrition Crisis Looms as More than 39 Billion In-School Meals Missed Since Start of Pandemic (UNICEF and WFP, 2021); https://www.unicef.org/ press-releases/nutrition-crisis-looms-more-39-billion-school-mealsmissed-start-pandemic-unicef-and

6. Barden-O'Fallon, J., Barry, M. A., Brodish, P. \& Hazerjian, J. Rapid assessment of Ebola-related implications for reproductive, maternal, newborn and child health service delivery and utilization in Guinea. PLoS Curr. https://doi.org/ 10.1371/currents. outbreaks.0b0ba06009dd091bc39ddb3c6d7b0826 (2015).

7. Laborde, D., Martin, W., Swinnen, J. \& Vos, R. COVID-19 risks to global food security. Science 369, 500-502 (2020).

8. Laborde, D., Smaller, C. \& Porciello, J. What Would it Cost to Avert the COVID-19 Hunger Crisis? (Cornell University, 2020); https://hdl.handle. net/1813/70172

9. Malpass, D. COVID Crisis is Fueling Food Price Rises for World's Poorest (World Bank Group, 2021); https://blogs.worldbank.org/voices/covid-crisisfueling-food-price-rises-worlds-poorest?cid=SHR_BlogSiteEmail_EN_EXT

10. Hamadani, J. D. et al. Immediate impact of stay-at-home orders to control COVID-19 transmission on socioeconomic conditions, food insecurity, mental health, and intimate partner violence in Bangladeshi women and their families: an interrupted time series. Lancet Glob. Health $\mathbf{8}$, e1380-e1389 (2020).

11. Headey, D. et al. Poverty and Food Insecurity During COVID-19: Telephone Survey Evidence from Mothers in Rural and Urban Myanmar (International Food Policy Research Institute, 2020); https://ebrary.ifpri.org/digital/ collection/p15738coll2/id/134036

12. Amare, M., Abay, K., Tiberti, L. \& Chamberlin, J. Impacts of COVID-19 on Food Security: Panel Data Evidence from Nigeria (International Food Policy Research Institute, 2020); https://ebrary.ifpri.org/digital/collection/ p15738coll2/id/133866

13. Kansiime, M. K. et al. COVID-19 implications on household income and food security in Kenya and Uganda: findings from a rapid assessment. World Dev. 137, 105199 (2021).

14. Egger, D. et al. Falling living standards during the COVID-19 crisis: quantitative evidence from nine developing countries. Sci. Adv. 7, eabe0997 (2021).

15. Wolle, A., Hirvonen, K., de Brauw, A., Baye, K. \& Abate, G. T. Household Food Consumption Patterns in Addis Ababa, Ethiopia Strategy Support Program Working Paper 139 (International Food Policy Research Institute, 2020); https://ebrary.ifpri.org/digital/collection/p15738coll2/id/133654
16. Ammar, A. et al. Effects of COVID-19 home confinement on eating behaviour and physical activity: results of the ECLB-COVID19 International Online Survey. Nutrients 12, 1583 (2020).

17. Harris, J., Depenbusch, L., Pal, A. A., Nair, R. M. \& Ramasamy, S. Food system disruption: initial livelihood and dietary effects of COVID-19 on vegetable producers in India. Food Secur. 12, 841-851 (2020).

18. Prioritize Health and Well-being Now and When Schools Reopen (UNESCO, 2020); https://en.unesco.org/news/prioritize-health-and-well-being-nowand-when-schools-reopen

19. Gentilini, U., Almenfi, M., Orton, I. \& Dale, P. Social Protection and Jobs Responses to COVID-19: A Real-Time Review of Country Measures (World Bank, 2020); https://openknowledge.worldbank.org/handle/10986/33635

20. Nishtar, S. Ehsaas Emergency Cash: a Digital Solution to Protect the Vulnerable in Pakistan During the COVID-19 Crisis (Government of Pakistan, 2020); https://www.pass.gov.pk/Document/Downloads/Ehsaas\%20Emergency \%20Cash\%20Report\%20Oct\%202020_Dec15_2020.pdf

21. Brinkman, H.-J., de Pee, S., Sanogo, I., Subran, L. \& Bloem, M. W. High food prices and the global financial crisis have reduced access to nutritious food and worsened nutritional status and health. J. Nutr. 140, 153S-161S (2010).

22. Carducci, B. et al. Food systems, diets and nutrition in the wake of COVID-19. Nat. Food 2, 68-70 (2021).

23. Roberton, T. et al. Early estimates of the indirect effects of the COVID-19 pandemic on maternal and child mortality in low-income and middle-income countries: a modelling study. Lancet Glob. Health 8, 901-909 (2020).

24. KC, A. et al. Effect of the COVID-19 pandemic response on intrapartum care, stillbirth, and neonatal mortality outcomes in Nepal: a prospective observational study. Lancet Glob. Health 8, E1273-E1281 (2020).

25. Martorell, R. Improved nutrition in the first 1000 days and adult human capital and health. Am. J. Hum. Biol. 29, e22952 (2017).

26. Shekar, M., Kakietek, J. J., Dayton, J. M. \& Walters, D. An Investment Framework for Nutrition: Reaching the Global Targets for Stunting, Anemia, Breastfeeding, and Wasting (World Bank, 2017); https://www.worldbank.org/ en/topic/nutrition/publication/an-investment-framework-for-nutritionreaching-the-global-targets-for-stunting-anemia-breastfeeding-wasting

27. Rytter, M. J. H., Kolte, L., Briend, A., Friis, H. \& Christensen, V. B. The immune system in children with malnutrition-a systematic review. PLoS ONE 9, e105017 (2014).

28. Prendergast, A. J. Malnutrition and vaccination in developing countries. Phil. Trans. R. Soc. Lond. B 370, 20140141 (2015).

29. Fore, H. H., Dongyu, Q., Beasley, D. M. \& Ghebreyesus, T. A. Child malnutrition and COVID-19: the time to act is now. Lancet 396, 517-518 (2020).

30. Black, R. E. et al. Maternal and child nutrition: building momentum for impact. Lancet 82, 372-375 (2013).

31. Protecting Maternal Diets and Nutrition Services and Practices in the Context of COVID-19 (UNICEF, Global Nutrition Cluster, GTAM \& WFP, 2020); https://www.ennonline.net/covid19maternalnutrition

32. De Pee, S. et al. Home fortification in emergency response and transition programming: experiences in Aceh and Nias, Indonesia. Food Nutr. Bull. 28, 189-197 (2007)

33. Das, J. K. et al. Preventive lipid-based nutrient supplements given with complementary foods to infants and young children 6 to 23 months of age for health, nutrition, and developmental outcomes. Cochrane Database Syst. Rev. 5, CD012611 (2019).

34. Stewart, C. P. et al. Lipid-based nutrient supplements and all-cause mortality in children 6-24 months of age: a meta-analysis of randomized controlled trials. Am. J. Clin. Nutr. 111, 207-218 (2020).

35. Hawkes, C., Ruel, M. T., Salm, L., Sinclair, B. \& Branca, F. Double-duty actions: seizing programme and policy opportunities to address malnutrition in all its forms. Lancet 395, 142-155 (2019).

36. Bhutta, Z, A. et al. How countries can reduce child stunting at scale: lessons from exemplar countries. Am. J. Clin. Nutr. 112, 894S-904S (2020).

37. Gillespie, S. \& van den Bold, M. Stories of change in nutrition: an overview. Glob. Food Sec. 13, 1-11 (2017).

38. Heidkamp, R. A. et al. Mobilising evidence, data, and resources to achieve global maternal and child undernutrition targets and the Sustainable Development Goals: an agenda for action. Lancet 397, 1400-1418 (2021).

39. COVID-19 and Nutrition: Impacts, Field Innovations, and the Way Forward (BIFAD, 2020); https://www.usaid.gov/sites/default/files/documents/ BIFAD - Covid_19 and Nutrition - Impacts Field_Innovations and the_Way_Forward_September_14_2020.pdf

40. Amjath-Babu, T. S., Krupnik, T. J., Thilsted, S. H. \& McDonald, A. J. Key indicators for monitoring food system disruptions caused by the COVID-19 pandemic: insights from Bangladesh towards effective response. Food Secur. 12, 761-768 (2020).

41. Adhikari, J., Timsina, J., Khadka, S. R., Ghale, Y. \& Ojha, H. COVID-19 impacts on agriculture and food systems in Nepal: implications for SDGs. Agric. Syst. 186, 102990 (2021) 
42. Abdoul-Azize, H. T. \& El Gamil, R. Social protection as a key tool in crisis management: learnt lessons from the COVID-19 pandemic. Glob. Soc. Welf. 2020, 1-10 (2020).

43. Laborde, D., Herforth, A., Headey, D. \& de Pee, S. COVID-19 pandemic leads to greater depth of unaffordability of healthy and nutrient-adequate diets in low- and middle-income countries. Nat. Food https://doi.org/10.1038/ s43016-021-00323-8 (2021).

44. Hawkes, C. \& Gallagher Squires, C. A double-duty food systems stimulus package to build back better nutrition from COVID-19. Nat. Food 2, 212-214 (2021).

45. Laborde Debucquet, D. \& Martin, W. Implications of the global growth slowdown for rural poverty. Agric. Econ. 49, 325-338 (2018).

46. World Economic Outlook, April 2021: Managing Divergent Recoveries (IMF, 2021); https://www.imf.org/en/Publications/WEO/Issues/2021/03/23/ world-economic-outlook-april-2021

47. Asian Development Outlook (ADO) 2020 Supplement: Paths Diverge in Recovery from the Pandemic (Asian Development Bank, 2020); https://www. adb.org/publications/ado-supplement-december-2020

48. Laborde, D., Martin, W. \& Vos, R. Impacts of COVID-19 on global poverty, food security, and diets: insights from global model scenario analysis. Agric Econ. 52, 375-390 (2021).

49. Headey, D. \& Ruel, M. Economic Shocks and Child Wasting IFPRI Discussion Paper 01941 (IFPRI \& CGIAR, 2020); https://ebrary.ifpri.org/digital/ collection/p15738coll2/id/133786

50. Brent, R. J. in Cost-Benefit Analysis and Health Care Evaluations 2nd edn, 313-339 (Edward Elgar Publishing, 2014).

51. Hoddinott, J., Alderman, H., Behrman, J. R., Haddad, L. \& Horton, S. The economic rationale for investing in stunting reduction. Matern. Child Nutr. $\mathbf{9}$ 69-82 (2013)

52. Gold, M. R., Siegel, J. E., Russell, L. B. \& Weinstein, M. C. Cost-Effectiveness in Health and Medicine (Oxford Univ. Press, 1996).

53. Neumann, P. J., Ganiats, T. G., Russell, L. B., Sanders, G. D. \& Siegel, J. E. Cost-Effectiveness in Health and Medicine (Oxford Univ. Press, 2016).

54. Horton, S. \& Ross, J. The economics of iron deficiency. Food Policy 28, 51-75 (2003).

55. Stuckler, D., Basu, S., Wang, S. W. \& McKee, M. Does recession reduce global health aid? Evidence from 15 high-income countries, 1975-2007. Bull World Health Organ. 89, 252-257 (2011).

56. Pearson, R. et al. Optima Nutrition: an allocative efficiency tool to reduce childhood stunting by better targeting of nutrition-related interventions. BMC Public Health 18, 384 (2018).

57. Optima Nutrition User Guide (Optima Consortium for Decision Science, 2019); http://optimamodel.com/docs/Optima\%20Nutrition\%20User \%20Guide\%20Feb2019.pdf

58. Scott, N. et al. Ending malnutrition in all its forms requires scaling up proven nutrition interventions and much more: a 129-country analysis. BMC Med. 18, 356 (2020)

\section{Acknowledgements}

This paper was written on behalf of the Standing Together for Nutrition Consortium, a multidisciplinary consortium of nutrition, economics, food and health system experts, currently consisting of 51 individuals from 32 organizations in high-income countries and LMICs, working to address the scale and reach of COVID-19-related nutrition challenges (see the Supplementary Information section 'Standing Together for Nutrition Consortium members'). The authors thank A. de Vries and C. Kissick for support with manuscript preparation, T. Brown for a final editing of the manuscript and I. Brouwer, N. Covic, S. de Pee, C. Hawkes, A. Herforth, C. Levin and P. Menon for critical review of the final paper and for providing input relating to previous versions of the manuscript. D.H. was funded by the Bill \& Melinda Gates Foundation (BMGF) through the project Advancing Research on Nutrition and Agriculture Phase II (investment ID: OPP1177007). The work on this study was supported by a grant (2005-04728) from the Children's Investment Fund Foundation. R.E.B., N.W., R.H., A.F., J.K.A., N.S., A.S. and M.S. received financial support from the BMGF (grant INV-031078) for development of the LiST and Optima tool and contributions to this research. Some of the underlying modelling used in this work has received indirect financial support from the US Agency for International Development, but the views and opinions expressed in this paper do not necessarily represent the views and opinions of the BMGF or US Agency for International Development.

\section{Author contributions}

J.K.A., R.E.B., D.H., R.H., S.O., M.R., N.S., M.S. and N.W. conceptualized and designed the study, performed the primary analyses and wrote and edited the manuscript. A.F., L.H., D.L., A.S. and M.T. contributed to conceptualization, analysis and editing of the manuscript. All authors reviewed and approved the final version of the manuscript.

\section{Competing interests}

The authors declare no competing interests.

\section{Additional information}

Supplementary information The online version contains supplementary material available at https://doi.org/10.1038/s43016-021-00319-4.

Correspondence and requests for materials should be addressed to S.O.

Peer review information Nature Food thanks Kenji Shibuya, Heather Brown and the other, anonymous, reviewer(s) for their contribution to the peer review of this work.

Reprints and permissions information is available at www.nature.com/reprints. Publisher's note Springer Nature remains neutral with regard to jurisdictional claims in published maps and institutional affiliations.

(c) The Author(s), under exclusive licence to Springer Nature Limited 2021 


\section{Reporting Summary}

Nature Research wishes to improve the reproducibility of the work that we publish. This form provides structure for consistency and transparency in reporting. For further information on Nature Research policies, see our Editorial Policies and the Editorial Policy Checklist.

\section{Statistics}

For all statistical analyses, confirm that the following items are present in the figure legend, table legend, main text, or Methods section.

n/a Confirmed

$\square \bigotimes$ The exact sample size $(n)$ for each experimental group/condition, given as a discrete number and unit of measurement

$\square$ \A statement on whether measurements were taken from distinct samples or whether the same sample was measured repeatedly

$\triangle$ The statistical test(s) used AND whether they are one- or two-sided

Х Only common tests should be described solely by name; describe more complex techniques in the Methods section.

Х $\square$ A description of all covariates tested

Х $\square$ A description of any assumptions or corrections, such as tests of normality and adjustment for multiple comparisons

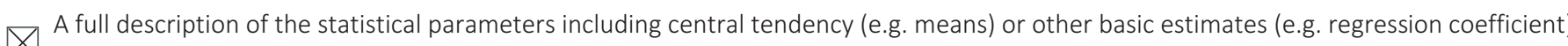

$\bigotimes$ AND variation (e.g. standard deviation) or associated estimates of uncertainty (e.g. confidence intervals)

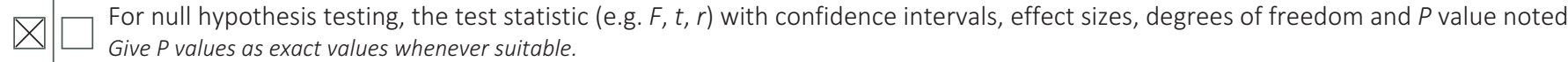

Х $\square$ For Bayesian analysis, information on the choice of priors and Markov chain Monte Carlo settings

$\square$ \ For hierarchical and complex designs, identification of the appropriate level for tests and full reporting of outcomes

Estimates of effect sizes (e.g. Cohen's $d$, Pearson's $r$ ), indicating how they were calculated

Our web collection on statistics for biologists contains articles on many of the points above.

\section{Software and code}

Policy information about availability of computer code

Data collection The study used secondary country level data.

Data analysis All software (modelling tools) used in the analyses of this study have been referenced before and are available here: (1)MIRAGRODEP: https:// www.ifpri.org/publication/miragrodep-model; (2) The Lives Saved Tool (LiST): https://www.livessavedtool.org/resources; (3) The Optima Nutrition model: https://optimamodel.com/nutrition/ .

For manuscripts utilizing custom algorithms or software that are central to the research but not yet described in published literature, software must be made available to editors and reviewers. We strongly encourage code deposition in a community repository (e.g. GitHub). See the Nature Research guidelines for submitting code \& software for further information.

\section{Data}

Policy information about availability of data

All manuscripts must include a data availability statement. This statement should provide the following information, where applicable:

- Accession codes, unique identifiers, or web links for publicly available datasets

- A list of figures that have associated raw data

- A description of any restrictions on data availability 


\section{Field-specific reporting}

Please select the one below that is the best fit for your research. If you are not sure, read the appropriate sections before making your selection.
Life sciences
Behavioural \& social sciences
Ecological, evolutionary \& environmental sciences

For a reference copy of the document with all sections, see nature.com/documents/nr-reporting-summary-flat.pdf

\section{Behavioural \& social sciences study design}

All studies must disclose on these points even when the disclosure is negative.

Study description

Research sample

Sampling strategy

Data collection

Timing

Data exclusions

Non-participation

Randomization
A modeling study using country-level data

We used country-level data of 118 low and middle income countries (LMIC, and listed in Supplementary Methodology 1) included in the Lives Saved Tool (LiST), with sufficient coverage data.

All analyses were performed on the same 118 LMIC included in LiST with sufficient coverage data (listed in Supplementary Methodology 1).

For the data collection in this study we used country-level data from three different modeling tools that have been described and referenced before: (1) MIRAGRODEP, a global computable general equilibrium model linked to country-specific household survey data (https://www.ifpri.org/publication/miragrodep-model); to link the outcomes of MIRAGRODEP to nutritional outcomes we used elasticities from a recent study that linked economic growth (GNI) shocks to child wasting and maternal low BMI risks to generate predicted changes in wasting and low BMI prevalence and referenced here: Headey D, Ruel M. Economic Shocks and Child Wasting [Internet]. Washington D.C.:; 2020. Report No.: IFPRI Discussion paper No 01941. Available from: http://ebrary.ifpri.org/utils/getfile/ collection/p15738coll2/id/133786/filename/133994.pdf. ; (2) the Lives Saved Tool (LiST), a mathematical modeling tool which allows users to estimate the impact of coverage change on nutritional outcomes and mortality in low and middle income countries ( https:// www.livessavedtool.org/resources); third the Optima Nutrition tool, a cohort model that includes includes an optimisation algorithm, which can be used to incrementally shift a fixed amount of funding between interventions until it achieves a budget allocation that maximizes (or minimizes) a given objective (https://optimamodel.com/nutrition/).

We used (secondary) country-level data as input in the analyses. The analyses were performed in September and October 2020.

We used country-level data as input in the analyses for Low- and Middle-Income Countries $(n=118)$ with coverage data on essential nutrition and health services

We used country-level data as input in the analyses

Participants were not allocated into experimental groups

\section{Reporting for specific materials, systems and methods}

We require information from authors about some types of materials, experimental systems and methods used in many studies. Here, indicate whether each material, system or method listed is relevant to your study. If you are not sure if a list item applies to your research, read the appropriate section before selecting a response.

Materials \& experimental systems

\begin{tabular}{|c|c|}
\hline $\mathrm{n} / \mathrm{a}$ & Involved in the study \\
\hline$\checkmark$ & $\square$ Antibodies \\
\hline$\triangle$ & $\square$ Eukaryotic cell lines \\
\hline$\bigotimes$ & $\square$ Palaeontology and archaeology \\
\hline$\bigotimes$ & $\square$ Animals and other organisms \\
\hline Х & $\square$ Human research participants \\
\hline$\bigotimes$ & $\square$ Clinical data \\
\hline$\bigotimes$ & Dual use research of concern \\
\hline
\end{tabular}

\begin{tabular}{l|l}
\multicolumn{2}{l}{ Methods } \\
\hline n/a & Involved in the study \\
$\bigotimes$ & $\square$ ChIP-seq \\
$\searrow$ & $\square$ Flow cytometry \\
$\square$ & $\square$ MRI-based neuroimaging
\end{tabular}

